\title{
La mujer y el personaje femenino en cuentos jordano-palestinos de tradición oral
}

\author{
La dona i el personatge femení en contes jordano-palestinians de \\ tradició oral
}

\author{
Women and female characters in Jordan-Palestinian tales from \\ the oral tradition
}

\author{
Margarita Isabel Asensio Pastor. Universidad de Almería. masensiop@ual.es \\ https://orcid.org/0000-0003-3182-2501
}

\section{Extended abstract}

This article aims to present a study on the presence of women and female characters in PalestinianJordanian tales from oral tradition and their didactic use from an intercultural and coeducational perspective. It is based on the ethnographic research carried out by Asensio (2015) through which she compiled a corpus of oral stories through a publication process. These stories were originally told in Arabic and were transcribed into Latin characters and translated into Spanish. It is understood that, being oral tales, they are presented in dialectal Arabic; specifically, in Jordan we can highlight four dialect varieties that respond to ethno-national origin (Transjordan / Palestine) and lifestyle (sedentary [urban, rural] or Bedouin [nomadic, semi-nomadic]. However, my interest in these stories arose because oral narrative is the most spread literary subgenre within Children's and Young Literature. I consider oral tradition the most ingrained discursive essence of the human being from an early age and children can easily recognize it. Additionally, the story is understood as an ethological fact. Although it may present certain geographical peculiarities, it is still a cross-cultural manifestation: all human beings narrate, and especially stories; all human beings use oral tradition to present social warnings, transmit values, etc.; the folktales, in addition, share common structural and organizational basic elements, although with their own spatial-temporal and socio-cultural idiosyncrasies. On the other hand, analysing stories from an oral tradition other than the Spanish one answers the need to train future teachers to recognize cultural diversity (global and national) and the different contributions which can be found, around the world, to inform this diversity. In the case at hand, for instance, data reveal a type of active female character that also serves to dismantle stereotypes and prejudices.

Along the same lines, this work aims to collaborate in the construction of a broader and intercultural literary canon of Children's and Youth Literature in the light of the reality of many Spanish educational classrooms (and of the world in general), overcoming the dominant monocultural approach to education. We agree with Colomer (2005) with the fact that literature is a space for the formation of cultures, but also a place to meet others.

Focusing on the data of the analysis, we must say that it followed the proposal of Dundes (1964) and Günthner and Knoblauch (1995) based on a holistic analysis considering: the text, the texture and the context. Thus, we can conclude that the role that women occupy within the framework of the Palestinian-Jordanian oral narrative places her as the "active depository" of said tradition, following 
the terminology of Sydow (1965). The age variable is also a determining factor along with the gender. In addition, women are culturally associated to fiction, compared to man characters that are linked to reality. This means, from the narratological point of view, that women in the studied context have a greater repertoire of stories than men, as well as a more varied and open subject matter. The Jordanian-Palestinian woman, upon reaching maturity, associated with menopause (Rabadán, 2003) and motherhood (Bassiouney, 2009), gains status and, at the same time, frees herself from all sexual charges and certain social taboos. Additionally, women have no qualms about introducing eschatological or erotic elements into their stories while men do.

On the other hand, the analysed stories show a tendency to introduce female characters under three parameters: youth, beauty and marriage. However, female characters do not always have a passive role, although initially it may seem that way. There are more female characters found than in canonical Western tales and, above all, they are active. Sometimes, they are born as passive characters depending on the will of parents, especially fathers, but in the face of adversity take control of the situation and face it without waiting to be saved by a typical "prince charming" of Western stories. Regarding the latter, it is observed that their active role has its reward, the power to marry a prince who appears to be passive and without any will since it is his mother who decides that marriage. Culturally, in the studied context, marriage is still valid as part of personal fulfilment, but it is not exclusive of the female gender. It is also a male goal and, consequently, it leads to procreation. The data also highlight the feminine role of mothers as the wise characters, who put order, who seek and recognize their lost daughters or who make decisions such as marriage as previously mentioned. The partial or total absence of the father figure is also interesting.

In short, the results of this research provide cultural and literary information for training Early Childhood and Primary Education future teachers from an intercultural paradigm and taking into account the gender issue. In the specific case of the analysed corpus and its context, it helps to confront existing stereotypes about Arab-Islamic culture, specially, cultural and gender stereotypes. This study offers an experience research to the lectures on Children's Literature useful for training students that can apply this knowledge in the school and, above all, can consider different sociocultural realities in order to work towards coeducation.

Finally, I hope this research contributes to expand the literary canon of Children's and Youth Literature from an intercultural and coeducational perspective. A canon, on the other hand, which encourages the inclusion of the active female character on an equal basis with males that serve to build more egalitarian and equitable societies in line with goal 5 of The 2030 Agenda for Sustainable Development of the United Nations (United Nations, 2015).

Key words: women, female character, folktales, Arabic oral tradition, coeducation, children's and youth literature

\section{Resumen}

Este artículo tiene como objetivo presentar un estudio sobre la presencia de la mujer y del personaje femenino en cuentos palestino-jordanos de tradición oral. Para ello, partimos de la investigación etnográfica realizada por Asensio (2015) y el corpus de cuentos por esta recopilados. Así, mostraremos, por un lado, el papel que ocupa la mujer dentro del marco de la narración oral como depositaria activa de dicha tradición siguiendo la terminología de Sydow (1965) y, por el otro, ofreceremos un estudio sobre el personaje femenino teniendo en cuenta el corpus mencionado. Estos datos servirán para dar a conocer nuevos contextos literarios para la construcción de un canon literario de LIJ intercultural, etnopluricultural y coeducativo para docentes de Educación Infantil y Primaria.

Palabras clave: mujer, personaje femenino, cuentos, tradición oral árabe, coeducación, literatura infantil y juvenil (LIJ) 


\section{Resum}

Aquest article té com a objectiu presentar un estudi sobre la presència de la dona i del personatge femení en contes palestinojordans de tradició oral. Per a açò, vàrem partir de la investigació etnogràfica realitzada per Asensio (2015) i el corpus de contes recopilats per aquesta. Així, mostrarem, d'una banda, el paper que ocupa la dona dins del marc de la narració oral com a dipositària activa de la dita tradició tot seguint la terminologia de Sydow (1965) i, d'una altra, oferirem un estudi sobre el personatge femení tenint en compte l'esmentat corpus. Aquestes dades serviran per donar a conéixer nous contexts literaris per a la construcció d'un cànon literari de LIJ intercultural, etnopluricultural i coeducatiu per a docents d’Educació Infantil i Primària.

Paraules clau: dona, personatge femení, contes, tradició oral àrab, coeducació, literatura infantil i juvenil (LIJ)

\section{Introducción}

La necesidad de construir sociedades más igualitarias en términos de género, equitativas y también respetuosas con otras culturas, hace que tengamos en cuenta el potencial de los cuentos de tradición oral para la formación de los futuros y las futuras docentes de Educación Infantil y Educación Primaria desde la coeducación y la educación intercultural.

En el mundo globalizado en el que nos encontramos, donde la relación con la "otredad" entendida como los grupos sociales culturalmente distintos al nuestro, debería ser más fluida a tenor de las posibilidades tecnológicas de las que disponemos para interconocernos como es Internet, lo cierto es que parece que aumentasen los malentendidos culturales, las incomprensiones, la estereotipación, los prejuicios y, en definitiva, los bulos y los discursos de odio, especialmente en las redes sociales (Campos, 2015; Cabo y García, 2017; Fundación Cepaim, 2019). De igual modo, siguen existiendo planteamientos asimétricos entre los distintos géneros asumiendo la tradicional binariedad de estos (masculino-hombre/femenino-mujer) y, por otro lado, los roles que hemos ido construyendo a tenor de esta dualidad en la que persiste la desigualdad. Esto hace que sean necesarios estudios que den cuenta de otros contextos culturales como es el caso de este artículo, centrado en cuentos de tradición oral jordanopalestinos y que lo hagan, además, desde una perspectiva de género alineados con la Agenda 2030 para el Desarrollo Sostenible de las Naciones Unidas, en concreto, con el objetivo 5, Igualdad de género (Naciones Unidas, 2015).

Por otro lado, en este trabajo nos hemos interesado por el cuento por ser el subgénero narrativo y literario de mayor difusión dentro de la Literatura Infantil y Juvenil (LIJ). El hecho de que 
proceda de una tradición oral distinta a la española se fundamenta en la necesidad de constituir un canon literario de LIJ más amplio e intercultural a tenor

El hecho de que proceda de una tradición oral distinta a la española se fundamenta en la necesidad de constituir un canon literario de LIJ más amplio e intercultural a tenor de la realidad de muchas aulas educativas españolas superando el enfoque monocultural dominante. de la realidad de muchas aulas educativas españolas superando el enfoque monocultural dominante. La literatura es un espacio de formación de culturas, pero también de encuentro con otras (Colomer, 2005) y es importante analizar el tratamiento que tiene la mujer y lo femenino en estas.

Así, la estructura expositiva que hemos seguido en este artículo es la que sigue: una justificación de la necesidad de atender al estudio de los cuentos de tradición oral y caracterización de los mismos; una aproximación a la tradición oral árabe seguida del contexto de investigación y la construcción del corpus de estudio; posteriormente, nos detenemos en el análisis propiamente dicho desde dos perspectivas: el contexto y el texto; al final, cerramos este artículo con las conclusiones donde incluimos el aprovechamiento de los datos obtenidos desde el punto de vista de la educación literaria con perspectiva de género intercultural.

\section{2. ¿Por qué los cuentos de tradición oral?}

Todos los pueblos establecen unos sistemas de transmisión cultural, una herencia narrativa a través de la cual, aprenden, aprehenden y comprenden el mundo, a sí mismos y a su comunidad (Calsamiglia y Tusón, 2001, p. 270). Así, narrar es un instrumento personal, social y cultural en donde el cuento ocupa un lugar privilegiado entre las diferentes manifestaciones narrativas desde temprana edad (Bassols y Torrent, 2003). Dentro de la narración destaca el cuento como un hecho transcultural, un hecho etológico en tanto que no parece tener un origen en la autorreproducción cultural, sino como una disposición mental más general, asumiendo los presupuestos de Schaeffer $(2005$, p.63). En este sentido, recogemos las palabras de la profesora Morote (2019):

El cuento oral es patrimonio de la humanidad y universal por naturaleza. Encontramos cuentos en todos los continentes y países. Unos se han trasmitido de generación en generación y luego se han fijado por escrito, y otros solo por escrito. Todos permanecen sin obstáculos de lenguas e ideologías durante el transcurrir de los tiempos. (p. 207). 
Además, el cuento de tradición oral comparte una serie de características que corroboran dicha

Dentro de la narración destaca el cuento como un hecho transcultural, un hecho etológico en tanto que no parece tener un origen en la autorreproducción cultural, sino como una disposición mental más general, asumiendo los presupuestos de Schaeffer. transculturalidad, exposición que sigue los presupuestos de Morote (2002), Pedrosa (2003) y Asensio (2015), entre otros. Por un lado, estos cuentos no tienen un autor 0 autora concreto/a, son anónimos, por tanto, son patrimonio de toda la comunidad. Por otro lado, son breves debido a que se apoyan en la memoria del transmisor o la transmisora. Esto último hace que empleen fórmulas hipercodificadas que funcionan como apoyos mnemotécnicos. Además, todos ellos responden a una estructura que el niño/la niña reconoce fácilmente y que tradicionalmente se sintetiza en introducción, nudo y desenlace, aunque Adam (1992, p. 57) concretó en el esquema de secuenciación narrativa que sigue:

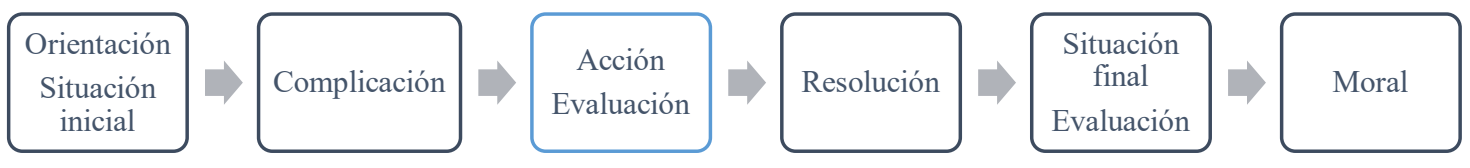

Gráfico 1. Secuencia narrativa de Adam (1992).

Asimismo, dispone de una temática basada en universales humanos que responden a las necesidades, sentimientos o emociones de los seres humanos como son el amor, la felicidad, la tristeza, la precaución, la envidia, etc. Sin embargo, no podemos olvidar que estas temáticas están condicionadas por los aspectos socioculturales y lingüísticos de la comunidad en las que se desarrollan. Por tanto, los cuentos de tradición oral contienen determinados mensajes endoculturales que sirven para conocer normas del grupo, moralidad o comportamiento de este (Gómez, 2002, p. 178).

Desde el punto de vista narrativo, es frecuente que los personajes se presenten como una categoría funcional (héroe/heroína; villano/a; ayudante; etc. (la tipología más conocida es la propuesta por Propp (1998), aunque resulta limitada para el cuento oriental como indica Mamet-Michalkiewicz (2011) en relación a las Mil y una noches), o como arquetipos y estereotipaciones en donde se juega con el dualismo bueno/a-malo/a; protagonista-antagonista y la presencia de un narrador omnisciente que es el que nos guía por el relato aportándonos los datos necesarios para reconocer el espacio, el tiempo o las emociones. También es frecuente 
que los cuentos combinen realidad y fantasía (verosimilitud e inverosimilitud). Como señalan Díez y Díez-Taboada (1999), en el cuento cabe todo:

\footnotetext{
lo real, lo maravilloso, la enseñanza y la diversión, lo trágico y lo cómico, lo cotidiano y el ensueño misterioso, el mundo infantil y el adulto, el amor y el odio, la crueldad y la bondad, la venganza y la generosidad (p.104).
}

Centrándonos en los personajes, los cuentos tradicionales pueden agruparse bajo un parámetro binario: hombre-niño (género masculino) /mujer-niña (género femenino). Al respecto, Holbek (1998) indica que todos los cuentos maravillosos son de dos tipos, masculinos o femeninos, y que tienen dos figuras principales, un héroe y una heroína; la inclusión en uno u otro tipo depende del grado de actividad del héroe/la heroína.

Ahora bien, Sanfilippo (2019) nos recuerda que gran parte de los cuentos de tradición oral que se conservan, fueron recogidos por hombres por lo que la tendencia dominante en los estos es la de plasmar aquellos de tipo masculino. En este sentido, como argumenta Taggart (1990), el género del narrador y la audiencia condiciona el argumento y el rol de los personajes principales. Por otra parte, la mayoría de los recopiladores eran hombres, de manera que las narradoras probablemente no les contarían ciertas historias reservadas a contextos exclusivamente femeninos Sanfilippo (2019).

Contar un cuento es un acto comunicativo complejo que no consiste simplemente en trasmitir información, sino que tiene la capacidad de sintetizar la visión y la organización del mundo (Sanfilippo, 2019). Además, al contar un cuento los niveles de producción en los que textura, texto y contexto (Dundes, 1964) se relacionan con la formulación, narración y dramatización de este. Precisamente, es en el acto narrativo en donde se ubican un compendio de valores naturales, sociales y culturales existentes en un lugar y un momento determinados, que influye en la vida material y psicológica del individuo en su comunidad y, a la vez, lo condicionan.

\section{Contextualización}

\subsection{Aproximación a la tradición oral árabe}

La tradición oral ha estado siempre presente a lo largo del tiempo en el mundo árabe. Como explica Allen (2000), las leyendas y los relatos pre-islámicos se han filtrado en la tradición oral. Así sucede con historias como la de Maŷnūn Layla, los ayyām al-'arab -epopeyas o gestas- (que recogen las guerras tribales al estilo de nuestra época) o la sīra -la biografía del Profeta Muhammad y posteriores colecciones de relatos calificados de populares como la historia de los 
Banī Hilāl que cuenta la historia de Abu Zayd al-Hilālī, héroe árabe que representa el coraje y la fortaleza y que viajó de Oriente a Occidente, hacia Túnez, conquistando estas tierras para el Islam (Allen, 2000); las historias jocosas del pícaro Ŷoḥa o la célebre compilación Las mil y una noches que para el-Shamy (2004) comprende gran parte de los relatos de literatura tradicional árabe:

most of the narratives constituting Alf laylah wa laylah in the Arabic language are literary representations of traditional folktales. These tales may be assumed to have been in oral circulation at the time(s) the book was being cumulatively formulated, and may represent older versions of contemporary folktales (p.34).

También, la primitiva lírica árabe, la denominada poesía ŷahilī1, manifiesta su carácter oral, pues como afirma Adonis2 (1997) nació como canto, "es decir, surge como algo que se oye, y no se lee, como cántico no como escritura. Era el verbo y otra cosa que lo trascendía, pues trasladaba la palabra, y algo que la palabra, especialmente la escritura, es incapaz de traducir" (p. 123).

Como ha pasado en otros lugares del mundo y otras literaturas, también la tradición oral ha estado relegada a un segundo plano, cuando no al ostracismo.

Ahora bien, los movimientos nacionalistas e independentistas de los distintos países que conforman el mundo árabe, coincide con lo que en literatura árabe se ha dado en conocer con la Nahḍa (se entiende como Renacimiento o Despertar árabe a nivel cultural).

Este movimiento se originó y desarrolló a mediados del siglo XIX y principios del XX. Este despertar supuso el resurgir folclórico posiblemente como un mecanismo de reafirmación nacional e idiosincrática frente al colonizador en una doble dirección: la 'uruba (la arabidad) y la reivindicación de la identidad regional dentro del marco del concepto compartido anterior. En definitiva, la Nahḍa supuso una toma de conciencia y la revalorización de la herencia folclórica de cada país árabe. Además, la urgencia de recuperar las tradiciones y especialmente la cultura oral vehiculada por las lenguas vernáculas como apunta Fanjul (1977), especialmente en países considerados en vías de desarrollo como sucede con muchos de los países árabes:

El interés por el folklore reviste un cierto carácter revolucionario que en los países árabes había sido sistemáticamente marginado por evadirse, a la vez, de la cultura clásica o de los modelos extranjerizantes. El surgimiento a flor de agua del folklore no

\footnotetext{
${ }^{1}$ Si bien el término es despectivo, significar "ignorante" en tanto que es anterior a la revelación coránica.

2 Pseudónimo con el que se conoce al poeta y ensayista sirio 'Alī Aḥmad Sa’îd Isbir (Esber).
} 
es tanto el del individuo como el de las clases desdeñadas. Porque aunque no exista una prohibición formal de publicar obras en árabe vulgar (el único que utiliza la literatura popular), sí existe una "selección por el descrédito" (p. 49).

Cabe también mencionar que, la recuperación de lo folklórico también ha estado unido a la consideración de las lenguas vernáculas árabes como portadoras de cultura, puesto que la 'cultura oficial árabe' se basa en la escritura y esta solo puede ser en árabe clásico ${ }^{3}$. Así, para Badawi (1992) la incursión de lo tradicional y popular en la esfera literaria supuso la ruptura de la barrera entre los dos registros lingüísticos; se rompen las barreras entre lo coloquial y el árabe clásico o estándar y, al mismo tiempo, este último se ve enriquecido por las aportaciones de la tradición oral y adoptando sus técnicas:

\section{[...] the range of themes has widened, the language has developed new registers, in particular thanks to the breaking down of barriers between colloquial and fușhà, the possibilities for enrichment offered by the Arabic oral and written literary tradition have been recognized, the techniques used have become increasingly sophisticated (p. 269).}

En definitiva, la tradición oral árabe ha sobrevivido al paso del tiempo, al margen o en paralelo, de la cultura culta y escrita, gracias al interés social que despierta, a lo que esta aporta a la comunidad en la que se encuentra.

\subsection{El contexto de la investigación}

Jordania comprende un área geográfica de $89.328 \mathrm{Km}^{2}$ y se encuentra ubicado en el corazón del Oriente Próximo. Este país limita al norte con Siria, al nordeste con Iraq, al este y sur con Arabia Saudí y al oeste con Cisjordania. Para entender este país, su sociedad, sus tradiciones o, concretamente, sus cuentos de tradición oral, hemos de tener presente los acontecimientos históricos-políticos más recientes (al-Wer, 1999).

Por un lado, nos encontramos con una población en la que predominan dos grupos etnonacionales (palestinos y transjordanos) con minorías circasianas, chechenas o armenias (sin contar con la población actual refugiada iraquí o siria o los grupos de inmigrantes egipcios o

\footnotetext{
${ }^{3}$ Al respecto, hemos de recordar que la lengua árabe se debe entender desde los presupuestos de diglosia. Desde la perspectiva tradicional fergusionana, encontramos dos variedades del árabe: una cultura o variedad alta que sería el árabe clásico o fushà propia del registro formal y, sobre todo, de la escritura y otra variedad baja, que serían los dialectos, propia del registro coloquial, sin representación escrita. No obstante, la situación lingüística jordana resulta mucho más compleja que la división dicotómica fergusioniana de diglosia (Asensio, 2018).
} 
filipinos). Por otro lado, la forma y estilo de vida también determina el tipo de etnocuento y la propia lengua dialectal de transmisión:

- La población sedentaria distribuida en dos tipos de asentamiento: uno urbano y el otro rural.

- La población seminómada que solo se mueve dos veces al año a zonas acotadas para el pastoreo.

- La población beduina, es decir, nómada.

Asimismo, como señala Asensio (2015), en Jordania resultan fundamentales dos tipos de agrupaciones sociales: la tribu, sobre todo entre población transjordana-beduina, y la familia extendida que podemos entender como una forma reducida de organización tribal en la que se engloba tanto al núcleo familiar propiamente dicho (padre-madre-hijos/as), como también a abuelos/as, tíos/as, primos/as, sobrinos/as, etc. y que sirve de soporte económico. Además, existe la tendencia a vivir todos en el mismo edificio o en el mismo barrio, estableciendo una cercanía también espacial. En concreto, los hijos varones casados tienden a vivir o en el mismo edificio de los padres o cerca de estos.

Por último, la religión oficial de este país (y preponderante) es el islam de rito sunní y escuela jurídica hanafí y representa el $97,2 \%$ de la población, con un $2 \%$ de chiíes y drusos. Otro $2,2 \%$ de la población es cristiana (tanto oriundos de palestina como transjordanos) en su mayoría griegos ortodoxos, aunque también encontramos maronitas, griegos y romanos católicos, sirios ortodoxos, coptos ortodoxos, armenios ortodoxos, protestantes y un $0,7 \%$ de personas que profesan otras religiones (CIA, en línea).

\section{La construcción del corpus de estudio}

Este estudio parte de un corpus de cuentos jordano-palestinos que forman parte de la tesis doctoral de Asensio (2015). Realizó una investigación de enfoque cualitativo basado en el trabajo de campo, en concreto, de corte etnográfico y en la que se emplearon, además de una revisión de la literatura, otra serie de instrumentos de investigación: la observación participante, la entrevista y el diario de campo.

Se construyó un corpus total con 27 cuentos de un total de 15 informantes. Estos fueron clasificados según el catálogo ATU (2004), en los casos en los que se encontraron equivalentes, como puede verse en el cuadro que sigue: 
Cuentos de animales. El hombre y los animales

Cuento 1: ar-raŷul al-ġabī w-at-ta'aleb makkār El hombre tonto y el zorro astuto [ATU 155]

Cuentos maravillosos

Cuento 2: bint al-armala. La hija de la viuda [ATU 312]

Cuento 3: al-g்ula wa-l-walad al-mu'addab. La ogresa y el niño respetuoso [ATU 110-312$327 / 327 \mathrm{~b}]$

Cuento 4: al-mihran al-ajzar. El caballito verde. [ATU 444]

Cuento 5: al-jayțān as-sa'ab. Los siete hilos

Cuento 6: ehdedwan wa ijwanoh. Ehdedwan y sus hermanos [ATU 124]

Cuento 7: el-g்ōl wal-awlād muš na īmīn. El ogro y los niños que no duermen [ATU 327C]

Cuento 8: 'ușșa leīla w-ạ̣-ḍīb. Leyla y el lobo [ATU 333]

Cuento 9 a: $\hat{Y}$ ubēna. Quesito [ATU 407]

Cuento 9 b: versión de Ŷubēna [ATU 407]

Cuentos de tontos o bobos

Cuento 10: ŷohā wal-lișș. Ŷoha y el ladrón [ATU 1341]

Cuento 11: ŷohā wal-armala. Ŷoha y la viuda [ATU 1341]

Cuento 12: ŷoha w martoh sagatat min-al-ḥmār. Ŷoha y su mujer, la cual se cayó del burro [ATU 1542]

Cuento 13: musmar ŷoha, El clavo de Ŷoha [ATU 1341]

Cuento 14: el-jēt al-aḥmar. El hilo rojo [ATU 1457]

Cuentos morales

Cuento 15: al-mustaqriḍāt Las prestadas. [ATU 294]

Cuento 16: kalba baraqiš. La perra Baraqiš.

Cuentos acumulativos

Cuento 17: al- 'otța w- dēlah. La gata y su cola [ATU 2034]

Cuento 18: dubbāna susu. La mosca Susu [ATU 2034]

Cuentos en los que se demuestra el ingenio de las personas

Cuento 19: bint al-șultān. La hija del sultán [ATU 875D]

Cuento 20: kēd an-nisā'. Las argucias de mujer [ATU 910]

Cuento 21: šēj al-'arab al-awal. El primer sabio (jeque) árabe [ATU 1542]

Cuento 22: el hallaq. El barbero [ATU 1542]

Cuentos de formulas

Cuento 23: giş̦̦a brid gazet. La historia de la jarra de aceite [ATU 2300]

Cuentos de matrimonios

Cuento 24: el-fuqarā'w-l-ḥubb. Los pobres y el amor.

Cuento 25: al-fustān. El vestido.

Cuento 26: al-ojtēin mutazwiŷāt w ŷuzhēin. Las hermanas casadas y sus maridos. 
En última instancia, procedimos al análisis del etnomaterial siguiendo el modelo propuesto por Dundes (1964) y Günthner y Knoblauch (1995) en tres niveles de análisis:

- La «textura», que es el aspecto formal de la actualización del mensaje. Hace referencia al nivel lingüístico y estilístico.

- El «texto», que es la versión individual recogida y relacionado con el nivel semántico. Atiende al contenido de los relatos en cuanto al tema, el argumento o los personajes.

- El «contexto» que son las situaciones sociales específicas en que se produce el hecho folclórico. Asimismo, contempla otros aspectos del acto narrativo como son los elementos no verbales y paralingüísticos.

\section{El análisis del corpus}

\section{1 Ámbito y modalidad de la transmisión del corpus}

La experiencia etnográfica nos hace situar la narración de cuentos de tradición oral en Jordania como un tipo discursivo propio del espacio privado:

- El cuento lo narra un miembro a otro de su propio núcleo familiar -incluida la familia extensa- o bien en el seno de la tribu.

- Jordania no dispone actualmente de narradores profesionales llamados hakawātī (حكواتي) o rāwī (راوي).

El orden social jordano se basa, como se expuso anteriormente, en dos sistemas superpuestos, la tribu y la familia extensa, y ambos núcleos constituyen el verdadero punto de inferencia cultural. Si bien el sistema tribal todavía pervive en la organización socio-política de Jordania, en realidad es la familia el ámbito más propio de la cultura asociada con lo popular, es decir, al margen o en paralelo, si se prefiere, de la cultura aprendida de tipo oficial e institucional más propia de la instrucción escolar (Asensio, 2015).

La familia es el primer grupo de relación del individuo y de transmisión cultural pues es un recipiente de la herencia filogénica, es decir, lo biológico y la ontogenia que es la cultura de cuya transmisión es garante. En la familia es donde el individuo primeramente interioriza normas, usos, costumbres, tradiciones y, también, valores, emociones, afectos, pensamientos, creencias, actitudes sociales.

Los cuentos que conforman este corpus están relacionados directamente con la familia y de forma directa o indirecta los informantes hacen mención al hogar como espacio narrativo: 
Ejemplo 1. Informante IHJ2: "ha-ḥikayə gallatnī emmī, ya'nī, sittī, ŷaddatī4 'ašān hiyyə sakinə ma'a ahlī]" ("Esta historia me la contó mi madre [o sea, mi abuela (repite la palabra abuela pero en árabe normativo) porque ella vive con mi familia)".

En este caso, además, enfatiza el hecho de que la abuela viviera con ellos.

Ejemplo 2. Informante IMJ1: "kāna eḥkilnā el-ŷiddāt | ygūlna w ummī, allāh yarḥamiha, teḥkilnā" ("nos lo contaban los abuelos, nos los contaban y mi madre, que Dios la tenga en su gloria, nos lo contaba").

Ejemplo 3. Informante IMP2, que a diferencia de los anteriores ejemplos, se realiza en inglés: "before we was, | were, were youth we doesn't TV so:: she, tells us stories and there are so:: nices:: | we didn't sleep | without stories" ("antes nosotros era | éramos, éramos jóvenes y no teníamos tele, por tanto:: ella, nos contaba historias y son tan:: bonitas:: | nosotros no podíamos dormir ( $\sin$ historias")

La narración del cuento folclórico en el contexto jordano se inscribe en la esfera privada porque carece de espacios públicos para tal fin.

\subsection{La voz autorizada para la narración de cuentos de tradición oral en Jordania}

Sydow (1965) había establecido dos categorías de depositarios de la tradición oral en función de un rol y un status en términos sociológicos que cada miembro tiene dentro de la sociedad (Giner, 2010):

1. El depositario activo que es aquel individuo que dentro de su grupo social mantiene, reproduce o produce tradición oral. Este puede ser un profesional de la narración o un aficionado. Dispone de unas características concretas, en otras palabras, de la 'competencia folclórica' suficiente entendida como "saber decir, saber escuchar, saber hacer, donde se ponen en juego las relaciones de comunidad consigo misma y con su entorno. Lo que se transmite con los relatos es el grupo de reglas pragmáticas que constituye el lazo social" (Lyotard, 1984, p. 48).

2. El depositario pasivo que conoce algunos materiales folclóricos, pero no suele ser trasmisor de forma activa. Para Taggart (1990), el género de los narradores y de la audiencia es determinante en tanto que condiciona el argumento, aunque hemos contemplado otras variables a la hora de estudiar al (la) narrador/a como son la edad, la educación y la

4 El informante emplea el término abuela en dialecto de tipo jordano que luego repite en árabe normativo. Esto se debe a un intento, por parte del informante, por explicar un vocablo que, a priori, no considera entendible por un extranjero. 
ocupación laboral. Todas estas variables influyen en la personalidad y la pragmática de la narrativa oral.

Así, el grupo final de informantes fue de 15 personas agrupadas en función de las variables edad, género, formación académica y procedencia etnonacional:

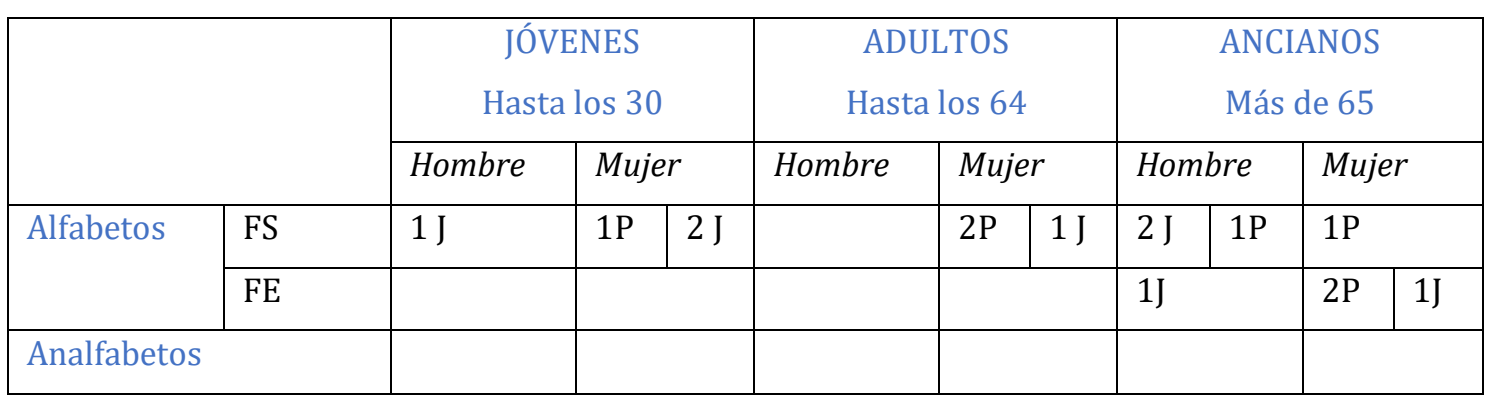

Cuadro 1. Variables sociales para la distribución de los informantes. ${ }^{5}$

En los epígrafes que siguen, nos vamos a detener en cada una de las variables indicadas.

\section{La variable de edad}

La edad de los/las informantes resulta significativa a la hora de determinar quién es o no es un depositario activo de la tradición oral.

En el caso de nuestro estudio, los informantes fueron distribuidos en tres grupos en función de esta:

- Jóvenes con una edad no superior a los 30 años. Se entrevistaron un total de 20 individuos estudiantes de español en la Universidad de Jordania y el Instituto Cervantes de Amán.

- Adultos cuyas edades oscilan entre los 31 y los 64 años.

- Mayores de 65 que es el grupo que cuenta con mayor número de informantes.

Del primer grupo, solo cuatro de ellos permanecen como narradores/narradoras al no considerarse a sí mismos narradores en contraposición a otros miembros de su familia como la madre, la abuela o los abuelos en general y, a veces, incluso tíos como se desprende de los siguientes testimonios de los informantes finalmente seleccionados:

\footnotetext{
${ }^{5}$ Leyenda FS = formación superior $/$ FE =formación elemental, $\mathrm{J}=$ Jordano $/ \mathrm{P}=$ Palestino.
} 
Testimonio 1. Informante IJM2. Esta parte de la intervención fue en español: "mi abuela es una experta en contar historias" "tengo una tía, la hermana de mi padre. Es mayor | no está casada, ella sabe contar muy bien".

Testimonio 2. Informante IHJ3. Para este informante, su abuela es el verdadero referente narrativo, así pues, afirma "mā ba'ref gușaș | ŷaddatī, na'm, hiyyə ta'ref" ("no sé cuentos | mi abuela, sí, ella sabe") y continúa diciendo "lamma kunt sḡîr, kunt dāiman ma'a ŷaddatī. hiyyə eḥkilnī gușaș dāìman | bas ensit kullu, [ya'nī] jallinī ufakkir šwai /" ("cuando era pequeño, estaba siempre con mi abuela. Ella me contaba cuentos siempre | pero olvidé todo, [bueno] déjame pensar un poco /").

Testimonio 3. Informante IMP3. El testimonio de esta informante es especialmente significativo porque no solo apuntó a su madre y a su abuela -que por el contexto se desprende que se trata de la abuela materna- como prototipos de depositarias activas, sino que insistió en que entrevistásemos a su madre (su abuela había fallecido). En efecto, su madre se convirtió en la informante IMP2. Esta informante IMP3 dijo lo siguiente: "tiḥkī ma'a ummī, hiyyə, na'm, hiyyə ta'ref hikayāt / wa allāh, ḥikayēt ŷamilə / (Ri) | habla con ella, yo, yo..- | ummī w teta /ahh..sameithum `ușaș ŷamilə | anā muš šatra li-I-ușāṣ bas:../ ummī wa ŷaddatī... mā ša` allāh! / | allāh yuharbaha/ | kanat amaizing | Leš ma tị̣ki ma'a ummī, profesora?/ ya'ni, ḥelwə ašan momkin usammi marra tānia al-ușaș li-eḥkilah li-awladī” ("habla con mi madre, ella, sí, ella sabe cuentos / por dios que sabe cuentos preciosos / (Ri) | habla con ella, yo, yo:: | mi madre y la abuelita / ahh- les escuché cuentos preciosos | Yo no soy muy lista para las historias [no sé] pero:.. mi abuela imadre mía! iqué Dios la tenga en su gloria! era increíble | ¿Por qué no hablas con mi madre, profesora? Bueno, genial ${ }^{6}$, porque podría escuchar otra vez las historias y contárselas a mis hijos").

La misma tendencia se observa en el grupo de informantes adultos. Para estos, también son sus mayores los narradores expertos y, por tanto, voces autorizadas como dice la informante IMP2: "bas what my mother said it much better than me | kîf!/ | and my grandmother (Ri) | sittī kān eḥkilnā muddalla, ba'dēn | mm:: | ba'dēn, kīf?, ah::, yalla | gèer teḥkillah, (Ri) aḥla minnī:.../ we always went to visit her to Nablus | she was amaizig:.., w-allāh, amaizing! / I remember her stories (Ri-MCa)" ("pero que era mi madre [que] decía esto mucho mejor que yo | icómo! / | y mi abuela (Ri) | nos contaba con cariño después | mm:: | después, ¿cómo?, ah::, venga |

\footnotetext{
${ }^{6}$ La informante utiliza el adjetivo helwə que significa literalmente "dulce", pero que puede ser traducido o bien como "bonito" o como "qué deleite / qué alegría".
} 
diferente la contaba (Ri) más bonita que la mía:.../ nosotros siempre íbamos a visitarla a Nablus | ella era increíble, ipor Dios, increíble!/ recuerdo sus historias (Ri-MCa)").

Por último, el tercer grupo también tiene como referentes narrativos de los cuentos folclóricos a sus mayores como se puede observar de las palabras de la informante IMJ1: "kāna ehkilnā elŷiddāt / ygūlna w ummī, allāh yarhamiha, teḥkilnā" ("nos lo contaban los abuelos, nos los contaban y mi madre, qué Dios la tenga en su gloria, nos lo contaba"). La diferencia de estos informantes con respecto al resto es que también dejan constancia de sus conocimientos como sucede con el informante IHJ1 cuando afirma "anā, ba'ref gușaș ktīr" ("yo sé muchas historias"). Por su parte el informante IHJ2, una vez que le expliqué los objetivos que pretendía alcanzar con este trabajo de investigación me dice "ḥelū/, ah::, 'anŷed /" ("iqué bonito!/ sí::, ide verdad!/") y comenzó con la primera historia, sin ningún preámbulo más. No justifican el origen de quién se lo contó -en muchos casos ni lo recuerdan-y no se plantean si son o no buenos narradores, sino que cuentan historias sin cuestionarse nada.

De lo expuesto se desprende que los depositarios activos de la tradición oral en el contexto de estudio son los miembros de mayor edad debido, a nuestro parecer, a cuatro motivos:

- La ausencia de tecnología. Así, por ejemplo, la informante IMP4 dice no recordar más historias porque las escuchaba en la radio: "mā tdakar / anà, kān 'indī radio" ("no recuerdo / yo, tenía radio").

- La educación, sobre todo para las niñas, era más limitada.

- El tiempo y la paciencia. La jubilación en Jordania es a los 60 años, siempre que las condiciones lo permitan, por lo que los y las jubiladas tendrán más tiempo para estar en el hogar con los más pequeños. Por otro lado, es más frecuente que las mujeres mayores hayan dedicado su existencia al hogar y la crianza por lo que continúan dicha labor con sus nietos. Generalmente, serán las abuelas paternas las que cuiden de los nietos/nietas de sus hijos varones. De igual modo, la cultura árabe en general suele mostrarse más paciente con los niños/las niñas por lo que se hace extensible a la narración de cuentos (Asensio, 2015).

- La memoria. En sociedades donde la oralidad es mayor, la memoria también. A esto se le une el mayor empleo de la memoria a largo plazo por parte de estos informantes.

No obstante, como afirma Sydow (1965), un depositario activo está supeditado al tiempo y al contexto, es decir, el informante-narrador cambia con el paso de los años porque se ve 
influenciado por sus experiencias personales, su capacidad memorística y actúa en función de la situación, el público (o el receptor al que se dirige). En definitiva, estoy de acuerdo con Lakoff (1972), para quien la experiencia vital es determinante porque la narración es la representación de vivencias pasadas.

\section{La variable de sexo y formación}

Si atendemos al género de los informantes, en palabras de Sadiqi (2003), el género en la narración adquiere significado. Para Sirhan (2014), referido a las historias de vida entre los palestinos expone: "there are notable differences in the personal narratives of woman and those of men, not only in style but also in content and form" (p. 222).

A este respecto, los introductores tendieron a ponerme en contacto con mujeres más que con hombres. Puede que por el hecho de ser mujer-investigadora. Como resultado, el $18,52 \%$ son narradores-hombres, frente al $81,48 \%$ que son mujeres-narradoras. ¿Qué es lo que motiva que la presencia femenina sea mayor que la masculina?

En primer lugar, la primera motivación se debe al ámbito de comunicación en el que se localizó este tipo de manifestación folclórica y que es el espacio privado. Si tenemos en cuenta los parámetros del contexto social jordano-palestino de índole patriarcal, en el que este espacio está reservado a la mujer. La mujer es considerada "el ángel del hogar", que es el lugar que tradicionalmente se le asigna y que, por extensión, la hace ser la encargada de educar, alimentar y vestir a los niños frente al hombre, que está asociado más bien a los espacios públicos.

En segundo lugar, el carácter ficcional del cuento folclórico tiende a relacionarse con la mujer. Rabadán (2003), al referirse a los cuentos de tipo fantástico, a los relatos maravillosos (aljrefiyye) en Palestina, considera que "es poco frecuente que los hombres narren este tipo de cuento indiscutiblemente relacionado con la patraña y la ficción (...)" por lo que los define como "un arte de mujeres" (p. 52). Los hombres entrevistados marcan más la barrera entre la imaginación y la realidad como lo demuestra el siguiente testimonio del informante IHJ3, el cual comienza su primera intervención narración advirtiendo de la veracidad del relato: “anā aḥkilek gișșa min ḥaqiqa | hada, hada, hada qișșa ḥaqīqī (Ri)" ("yo te voy a contar un cuento de verdad. Este (este, este) es un cuento verdadero"). 
Por último, y teniendo presente la relación que se ha establecido entre mujer y ficción, se halla otro aspecto que ha de tenerse en cuenta: la edad de la mujer. Esta, al alcanzar la madurez asociada a la menopausia (Rabadán, 2003) y a la maternidad (Bassiouney, 2009), gana estatus $y$, al mismo tiempo, se libera de toda carga sexual y de ciertos tabúes sociales por lo que no tiene reparos en introducir elementos escatológicos o eróticos en sus historias. Así pues, la informante IMJ1 narra el cuento C19 titulado Kēd al-nisā que trata de las argucias ${ }^{7}$ de las mujeres y previene contra ellas: "Gālloh: "Yā rab, yā rab taṣēr tad'ī la-rabha ennoh ŷōzha yen'amī w yiŷĩ șāḥhha illī hiyyə btaŷraf wiyāh w btat'alal hadā huwwə "hātī" hiyyə wiyāh w ennoh mā yšōfoh ("Señor mío, Señor mío... que su marido se hiciera ciego para que su amigo (amante) vaya a pasar tiempo con ella y para

[...] al alcanzar la madurez asociada a la menopausia (Rabadán, 2003) y a la maternidad (Bassiouney, 2009), gana estatus $y$, al mismo tiempo, se libera de toda carga sexual y de ciertos tabúes sociales por lo que no tiene reparos en introducir elementos escatológicos o eróticos en sus historias que su marido no lo vea"). No sucede lo mismo en el caso de los hombres que se presentan más pudorosos como en el ejemplo extraído del informante IHJ2 durante la narración del cuento C.6: "lāzim aḍhạak 'alayoh î-šī 'ašgla kabira [2=fī î-šī 'ușșa bilbațțīja bisāwī î-šĩ kān byḥ̂ī w lāî-šī=] ah:: huwwa | nāsī [2=lā, bibdanā hadōl al-qișaș, lāzim hadōl al-muhmīn=] șār yafir min al-bațțija ḥassabat nab'a, nab'a w tašrab tagūl: "la'nā allāh 'alayah mayya māliḥa" ("tengo que reírme de él haciendo algo grande. (=hay algo del melón, hace algo que::: que estaba llorando... [2=sí::, él | olvidé=] =no queremos historias así, son necesarias estas son las interesantes=] se puso a mear desde la sandía y ella creía que era una fuente (agua saliendo) y la bebió y dice "Dios maldiga a esta agua que está salada"); en cuanto a la presencia de lo erótico en el relato masculino debe observarse que este estará totalmente ausente cuando en su auditorio hay mujeres. Impera, en este caso, la restricción impuesta por el pudor (jayal-

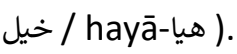

Herrero Muñoz-Cobo (1996: 118) observa en el caso tetuaní que la edad libera a las mujeres que emplean un discurso difesmístico. Para un occidental, desde el punto de vista de la imagen (Goffman, 1967 [1970]) podría calificarse de desinterés por su propia imagen, aunque en realidad, es una clara demostración del estatus adquirido con los años.

Una última observación relativa al sexo de los informantes se refiere a la variedad de la lengua con la que se comunicaron pues, exceptuando los casos de alumnas estudiantes de español y el

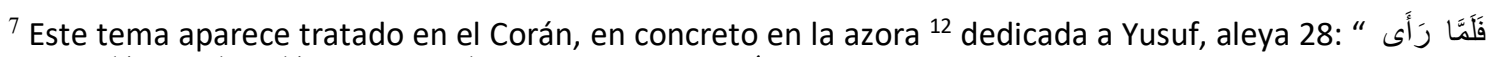

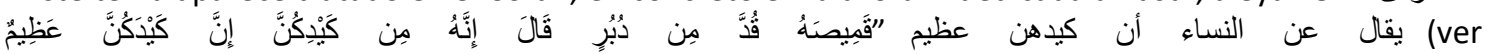
http://www.holyquran.net/cgi-bin/prepare.pl?ch=12)
} 
caso de la informante IMP2, por lo general, las mujeres emplearon el árabe dialectal en todo momento. Los hombres entrevistados muestran mayores reticencias al uso dialectal. En el caso de la informante IMJ1, debido a lo complicado de su variedad dialectal beduina, o bien sustituía alguna palabra en su variedad dialectal por otra estándar o recurría a su nieta para que esta la explicara en español. Por su parte, los hombres sí mostraron, como digo, más reticencias a la hora de emplear el árabe dialectal.

En cuanto a la formación de los/las informantes, existe una clara relación entre el sexo y la edad afectando a las mujeres como tendencia generalizada a nivel mundial, ya que el acceso a la educación era (y es en ciertas comunidades) más limitado para estas y, sobre todo, en la Jordania y Palestina de hace 40 años. En el caso estudiado destaca la informante IMJ1 por ser la que más etnomaterial transmitió y por su calidad narrativa. seguida de IMP4, IHJ1 e IMP1. En definitiva, como afirma Pinon (1965), "un buen informante debe poseer un lenguaje poético; una memoria excelente; cierta resistencia física y tener un estilo que agrade al auditorio" (p. 47). En el estudio de Asensio (2016) sobre el mundo árabe y los cuentos de tradición oral corrobora que la mujer sea"la voz autorizada y depositaria activa de la tradición oral árabe" (p.304) y donde libera su voz y su sabiduría.

\section{La variable estilo de vida y origen}

En cuanto a las variables estilo de vida y origen etnonacional, destacamos dos cuestiones, la primera es que existe un mayor número de narradoras mujeres palestinas de estilo sedentario urbano (IMP1, IMP2, IMP5 e IMP6) y que los hombres narradores son mayoritariamente transjordanos (IHJ1, IJH3 -sedentarios, urbanos- e IHJ2 (sedentario-rural).

Por otro lado, estas variables afectan también al aspecto lingüístico -la variable diatópica(Suleiman, 1985; Asensio, 2018) y desde el punto de vista del texto, es decir, la tipología de cuento y el argumento del mismo.

\subsection{Análisis de nivel textual}

Dentro de este nivel nos vamos a detener en dos aspectos concretos: el sexo del personaje (de los personajes) y la temática y caracterización de estos personajes.

\section{Estudio del personaje}

En los cuentos analizados destaca el hecho de que el personaje femenino sea porcentualmente superior al masculino, aunque el papel protagonista esté igualado. En concreto, aparece un $66,67 \%$ de mujeres, frente a un $25,73 \%$ que es íntegramente masculino. Por otro lado, hemos de aclarar que los cuentos que tienen a animales como personajes u objetos (que constituyen 
el 7,41\% de los cuentos), no han sido contabilizados en este cómputo. Si bien los tres aparecen en femenino (C16 kalba (perra), C17 'oțța (gata) y C18 dubbāna (mosca), esta última no marca el sexo porque sucede igual que en español, es decir, es un sustantivo epiceno.

En cuanto al papel activo o pasivo del personaje femenino dentro de los cuentos analizados, destacan dos cuestiones. Por un lado, la presencia de personajes femeninos secundarios activos (C4); se trata de la hija del sultán que es la que se ofrece

Destacan dos cuestiones. Por un lado, la presencia de personajes femeninos secundarios activos [...]Por otro lado, en los cuentos C2 y C5 se observa un fenómeno similar y es que inicialmente los personajes femeninos aparecen como pasivos en tanto que dependen de la voluntad de la madre (C2) o del padre (C5), pero luego se muestran activos. a investigar el misterio de las monedas de oro que aparecen cada noche en casa de un pobre, mientras desaparecen del castillo. Por otro lado, en los cuentos C2 y C5 se observa un fenómeno similar y es que inicialmente los personajes femeninos aparecen como pasivos en tanto que dependen de la voluntad de la madre (C2) o del padre (C5), pero luego se muestran activos. En el primer caso, la madre acuerda el matrimonio de esta con un forastero recién llegado al pueblo que busca casarse y en el segundo caso, el padre dispone que salgan por la noche de casa para, posteriormente, este la abandone de noche en mitad del desierto. Ambos personajes no quedarán a expensas de su situación: una movida por la curiosidad y la otra por la necesidad van a tener un papel reactivo. Así, la primera incumple la norma de su marido de no entrar en una habitación determinada del castillo. Con este incumplimiento sabe que su marido es en realidad un gul (equivalente a ogro) camuflado en la apariencia de un bello y apuesto hombre; esperará para poder escapar siendo perseguida por el gul y volverá a descubrirlo al transformarse este en cordero, si bien no será ella la que lo mate, sino el padre de esta -contradictoriamente, ya que al inicio aparece como hija de una viuda. En cuanto al personaje del cuento $\mathrm{C5}$, cuando esta se despierta y ve que no está el padre, no se quedará paralizada a esperar a ser rescatada, sino que tomará la iniciativa de salir del sitio en el que se encuentra y buscar una solución; tras varias aventuras, la protagonista terminará casándose con un príncipe.

En el caso de los cuentos C9a y C9b el personaje de Ŷubēna es el de una chica sumisa y obediente. Así, en el caso de la versión b, este personaje está a merced de la decisión familiar (no se especifica si del padre o de la madre) de casarla con un joven de otra tribu, aunque sabemos que es la madre la que pone una esclava negra para acompañarla en el viaje y que será esta esclava la que la suplante al tiznarla de negro. En la versión a, la envidia de las chicas del pueblo 
- que la dejarán colgada en una palmera- es lo que hará que la rescate un príncipe, aunque trabajará como una sirvienta al estar sucia.

En ambos cuentos, se oculta la belleza de la protagonista (sucia o tiznada de negro), y ambas pasan a ser pastoras al servicio del príncipe o de la tribu. En ambas versiones, la madre de Ŷubēna será la encargada de buscarla y reconocerla al oírla cantar. Una vez lavada, aflorará su belleza y blancura, por lo que la madre del príncipe (en ambos cuentos) decida casarla con su hijo.

Otro cuento, en este caso, el C26, muestra el papel determinante de la madre de las hermanas casadas, representando la justicia y la sabiduría. El cuento narra la envidia que siente una hermana por la otra por los regalos que le hace su marido. La madre es la que pondrá sentido a la situación al indicarle que ella tiene amor y la otra no.

El cuento C24 destaca por presentar una situación igualitaria entre el personaje masculino y el femenino. Se trata de un matrimonio que celebra su aniversario, pero desde la pobreza. Para poder hacerle un regalo al otro, cada uno de ellos vende algo importante: uno el reloj y la otra su pelo. La cuestión es que los regalos que se hacen tienen relación directa con estos objetos: él vende su reloj para comprarle una diadema y ella vende su hermoso pelo para comprarle a él una cadena para su reloj.

Un caso excepcional es el cuento C20 en donde un hombre le narra a otro la historia de una mujer que engañaba a su marido con otro amante. Si bien muestra un personaje femenino activo, en realidad lo hace como advertencia de lo que no debe hacer una mujer.

\section{Temática y caracterización del personaje femenino}

Sobre la temática, ya apuntamos en el epígrafe relativo al análisis contextual que la tendencia predominante es que la mujer narre historias con un mayor grado de ficción que las del hombre. No obstante, la otra cuestión a tener en consideración es que la mujer dispone de un repertorio mayor de cuentos de tradición oral que el hombre. Además, los personajes, sobre todo los/las protagonistas, de los cuentos narrados por mujeres no están sujetos al propio género de estas. Sin embargo, en el caso de los hombres, sí influye su género; así, la tendencia es contar historias en los que los personajes sean héroes masculinos activos. 
Cabe mencionar que suelen presentarse personajes femeninos que destacan por su belleza (C2, C5, C9a, C9b). Además, las protagonistas son

Sobre la temática, [...] la tendencia predominante es que la mujer narre historias con un mayor grado de ficción que las del hombre. No obstante, la otra cuestión a tener en consideración es que la mujer dispone de un repertorio mayor de cuentos de tradición oral que el hombre. mayoritariamente mujeres jóvenes (además de los cuentos anteriormente citados, también C8, C19). Solo en un caso se habla explícitamente de un personaje principal femenino viejo (C15). En el caso de los personajes masculinos, no aparecen atributos tales como belleza o edad que podemos intuir por la temática de los cuentos; en estos últimos se hace más hincapié en la sabiduría (C21), la inteligencia y valentía (C6) o en la estupidez-inteligente que está presente en el personaje de Ŷoḥa .

Con respecto a la temática, existe una tendencia a asociar lo femenino con el matrimonio lo que está presente en gran parte de los cuentos y su importancia, todavía hoy en día, en la sociedad árabe jordano-palestina. En concreto, en el tipo de repertorio compilado, aparecen tres cuentos que hemos agrupado bajo la categoría de matrimonio $(\mathrm{C} 24, \mathrm{C} 25, \mathrm{C} 26) \mathrm{u}$ otros donde aparece el protagonista casado como es el caso de Ŷoha (C12) o el cuento C20. También hemos de mencionar la presencia de mujeres jóvenes casaderas como en C2, C4, C5, C9a, C9b, C14, C19. En el caso del C14 se trata de chicas con un defecto en la pronunciación, por lo que la madre les indica que guarden silencio para que los hombres no lo noten y puedan casarse; y en el caso del C19, quien descifre el enigma de la hija del sultán, podrá casarse con ella. Por otro lado, aparece el matrimonio concertado en tres tiempos: al inicio de los mismos ( $C 2, C 9 a)$, en medio del relato (C4) -este cuento tiene la característica de ser acumulativo, es decir, presenta dos cuentos entrelazados por el caballito verde- $\mathrm{o}$ al final de estos, como premio (C9a, C9b, C5).

Por otro lado, no podemos olvidar, según los postulados de Propp (1998), que los personajes cumplen una determinada función en el relato, aunque no concuerden sus postulados funcionales con los cuentos orientales, ya que en estos encontramos heroínas mujeres (personaje activo), como ya expusimos. Además, es frecuente que estos personajes femeninos se presentan como arquetipos donde prevalecen la belleza (C9a, C9b, C2 y C5), la obediencia, (C9a, C9b, C8), la envidia (C9a, C9b,C26), el hecho de ser caprichosa, consentida o mimada (C19, C25) o su inteligencia para acometer lo que el C20 califica de argucias de mujer; en este sentido, el C20 asocia la astucia femenina como negativa y denunciada, dentro el propio cuento, por un personaje masculino. Sobre esta presentación de la mujer, lo encontramos también en el 
personaje de las sirvientas de los cuentos C9a y C9b o en el C5 que le hacen algo malo a la protagonista.

No obstante, la otra lectura que podemos hacer de lo femenino en este corpus -relevante para nuestro propósito- es que nos encontramos con mujeres valientes (C2 y C4) que coinciden con ser personajes resolutivos. Desde la perspectiva de la educación literaria, la introducción de este tipo de personajes en el aula de Infantil y Primaria permite propuestas de intervención didáctica coeducativas y, al mismo tiempo, interculturales.

Mención especial merece el papel de la madre en estos cuentos analizados. Por un lado, esta puede mostrar

Desde la perspectiva de la educación literaria, la introducción de este tipo de personajes en el aula de Infantil y Primaria permite propuestas de intervención didáctica coeducativas $\mathrm{y}$, al mismo tiempo, interculturales. preocupación por la situación de su hija, hecho determinante para que emprenda un viaje hasta encontrarla (C9a, C9b) o, por el contrario, puede ser la que resuelva el conflicto entre hermanas a través de su sabiduría como es el caso del cuento C26.

\section{Conclusiones}

El análisis de los etnocuentos que aquí presentamos muestra el lugar de la mujer árabe jordano-

El análisis de los etnocuentos que aquí presentamos muestra el lugar de la mujer árabe jordano-palestina dentro del hecho narrativo como la principal depositaria activa; el repertorio de estas suele ser mayor $y$ más variado que el de los hombres palestina dentro del hecho narrativo como la principal depositaria activa; el repertorio de estas suele ser mayor y más variado que el de los hombres. No obstante, en este aspecto no podemos descartar la condición de mujer de la propia investigadora, por lo que afectaría al repertorio narrado de las mismas de manera positiva, es decir, sentirse con mayor libertad a la hora de contar cualquier tipo de cuento. Además, la edad, en relación al sexo, es igualmente determinante en el caso de las mujeres-narradoras, puesto que las mujeres mayores se presentan libres de tapujos y convencionalismos sociales, a diferencia del hombre, más pudoroso e inhibido.

Por otro lado, los cuentos analizados manifiestan una tendencia a presentar al personaje femenino bajo tres parámetros: juventud, belleza y casadera. No obstante, no tienen siempre un rol pasivo, aunque inicialmente así pudiera parecer. 
Así, los resultados de esta investigación proporcionan una información cultural y literaria que puede servir para:

1. La formación de los futuros/futuras maestras/os de Educación Infantil y Primaria desde el paradigma intercultural y teniendo en cuenta la cuestión de género. En el caso concreto del corpus analizado y su contexto, confrontan los estereotipos que se tiene sobre la cultura árabe-islámica de tipo cultural y de género.

2. Ofrecer una experiencia de investigación en el aula universitaria de LIJ que sirva para que el alumnado en formación pueda aplicarla a su aula y, sobre todo, atendiendo a la realidad del contexto socio-cultural de esta desde la coeducación.

3. Ampliar el canon literario de la LIJ desde una perspectiva intercultural y coeducativa dando cabido, por otro lado, a la posible realidad de las aulas con alumnado extranjero o, nacidos en España, pero con progenitores extranjeros. Un canon, por otra parte, en el que se fomente la inclusión del personaje femenino activo y en régimen de igualdad con el masculino que sirvan para construir sociedades más igualitarias y equitativas en línea con el objetivo 5 de la Agenda 2030 para el Desarrollo Sostenible de las Naciones Unidad (Naciones Unidas, 2015).

\section{Referencias}

Adonis [Alī Aḥmad Sa'îd Isbir] (1997). Poesía y poética árabes. Madrid: Ediciones del Oriente y del Mediterráneo.

Agencia Central de Inteligencia estadounidense [CIA -Central Intelligence Agency] (en línea). Jordan. Recuperado de https://www.cia.gov/library/publications/the-world-factbook/geos/jo.html

Allen, R. M. A. (2000). An introduction to Arabic Literature. Cambridge: Cambridge University Press.

Adam, J. M. (1992). Les textes: types et prototypes. Récit, description, argumentation, explication et dialogue. París: Nathan.

Asensio Pastor, M. I. (2015). Cuentos jordanos de tradición oral: Estudio etnolingüístico y aplicaciones didácticas. [tesis doctoral]. Almería: Universidad de Almería.

Asensio Pastor, M. I. (2016). La voz femenina de la tradición oral. Raudem. Revista de Estudios de las Mujeres, (4), 290-306. Recuperado de http://ojs.ual.es/ojs/index.php/RAUDEM/article/view/1760

Asensio Pastor, M. I. (2018). La realización del fonema /q/ en las variedades dialectales del árabe en Jordania: análisis de cuentos de tradición oral. Estudios de Fonética Experimental, XXVII, 125-150. Recuperado de https://www.ub.edu/journalofexperimentalphonetics/pdf-articles/XXVII-09MAsensio-separata_new.pdf

Badawi, E. M. (1973). Mustawayāt al-'arabiyat $_{i}$ al-mu'āșirati fi mișr. El Cairo: Dār al-Ma'ārif.

Bassiouney, R. (2009). Arabic sociolinguistics. Edimburgo: Edinburgh University Press.

Bassols, M., y Torrent, A. M. (2003). Modelos textuales. Teoría y práctica. Barcelona: Octaedro. 
Cabo Isasi, A., y García Juanatey, A. (2017). El discurso de odio en las redes sociales: un estado de la cuestión. Barcelona: Ajuntament de Barcelona. Recuperado de https://cutt.ly/bgVbLkP

Calsamiglia Blancafort, H., y Tusón Valls, A. (2001): Las cosas del decir. Manuela de análisis del discurso. Barcelona: Ariel.

Campos, M., Ramos, M., Trejo Delarbre, R., Hernández Ramírez, M. E., De Dios Corona, S. R.,... Caballero Ochoa, J.L. (2015). Mensajes de odio y discriminación en las redes sociales. México D. F.: SEGOB; Consejo Nacional para Prevenir la Discriminación. Recuperado de https://cutt.ly/IgVnrRf

Colomer, T. (2005). Andar entre libros. México D. F.: Fondo de Cultura Económico.

Dundes, A. (1964). The Morphology of North American Indian Folktales. FF Communications, 195. Helsinki: Academia Scientiarum Fennica.

El-Shamy, H. (2004). Types of the Folktale in the Arab World: A Demographically Oriented Tale-Type Index. Bloomington: Indiana University Press.

Díez, M., y Díez-Taboada, P. (1999). Madrid: Espasa-Calpe.

Fanjul, S. (1977). Literatura popular árabe. Madrid: Editorial Nacional.

Fundación Cepaim (2019, noviembre 14). Ante el aumento del discurso del odio, más herramientas vivas para "Desactivar" prejuicios y estereotipos. Fundación Cepaim. Convivencia y cohesión social. Recuperado de https://cutt.ly/BgVnu8s

Giner, S. (2010). Sociología. Barcelona: Ediciones Península.

Goffman, E. (1967 [1970]). Ritual de la interacción. Ensayos sobre el comportamiento cara a cara. Buenos aires: Tiempo Contemporáneo.

Gómez López, N. (2002). Los géneros de la literatura de tradición oral: algunas proyecciones didácticas. Lenguaje y textos, 18, 175-181. Recuperado de https://ruc.udc.es/dspace/handle/2183/8159

Günthner, S. y Knoblauch, H. (1995). Culturally patterned speaking practtces-the analysis of communicative genres. Pragmatics, 5 (1), 1-32.

Holbek, B. (1998). Interpretación of Fairy Tales. Helsinki: Soumalainen Tiedeakatemian.

Lyotard, J. F. (1984). La condición postmoderna. Madrid: Cátedra.

Mamet-Michalkiewicz, M. (2011). Between the Orient and the Occident Transformations of The Thousand and One Night. Katowice: Wydawnictwo University Slaskiego.

Morote Magán, P. (2002). El cuento de tradición oral y el cuento literario: de la narración a la lectura. L. J. Tigero González (Coord.); A. Mendoza Fillola (Dir.) La seducción de la lectura en edades tempranas (pp. 159-197). Madrid: Secretaría General Técnica. Centro de Publicaciones. Ministerio de Educación, Cultura y Deporte.

Morote Magán, P. (2019). El valor universal del cuento. El español por el mundo, 2, 207-219. Recuperado de https://www.aepe.eu/revista/revista-n2/13_Pascuala_Morote_Magan.pdf

Naciones Unidas (2015). La Agenda 2030 para el desarrollo sostenible. Recuperado de https://cutt.ly/qgVmFtp

Oriol, C. (2017). Cuentos populares con protagonistas activas: la cara más desconocida de la tradición. En M. Sanfilippo, H. Guzmán y A. Zamorano (Coords.). Mujeres de palabra: género y narración oral en voz femenina. (pp. 17-32). Madrid: UNED.

Pedrosa, J. M. (2003). Los géneros de la literatura tradicional. En N. Gómez López, y J. M. Pedrosa (Eds.) Las voces son fronteras: didáctica de la literatura oral y la literatura contemporánea (pp. 1534). Almería: Universidad de Almería.

Pinon, R. (1965). El cuento folclórico. Buenos Aires: Universidad de Buenos Aires. 
Propp, V. (1998). Morfología del cuento. Madrid: Akal.

Rabadán Carrascosa, M. (2003). La jrefiyye Palestina: Literatura, mujer y maravilla. El cuento maravilloso palestino de tradición oral. Estudio y textos. México D.F.: Colegio de México.

Sadiqi, F. (2003). Women, gender and language in Morocco. Leiden y Boston: Brill.

Sanfilippo, M. (2019). Los cuentos maravillosos de las mujeres y las maravillosas mujeres de los cuentos. El AEDO. Revista del mundo de la narración oral, 9 (diciembre), 129-134.

Schaeffer, J.M. (2005). Adiós a la estética. Madrid: La balsa de la Medusa-Antonio Machado Libros.

Sirhan, N. (2014). Folk Stories and Personal Narratives in Palestinian Spoken Arabic: A cultural and linguistic study. Chennai: Palgrave Macmillan.

Suleiman, S. M. (1985). Jordanian Arabic Between Diglossia and Bilingualism: Linguistic Analysis. Amsterdam: John Benjamins Publishing Company.

Sydow, C. W. von (1965). Folktale Studies and Philology: Some Points of view. En A. Dundes (Ed.) The Stady of of Folklore (pp. 219-242). Englenwood Cliffs: Prentice-Hall.

Taggart, J. M. (1990). Enchanted Maidens: Gender Relations in Spanish Folktales of Courtship and Marriage. Princeton/New Jersey: Princeton University Press.

Wer, E. (1999). Why Do Different Variables Behave Differently? Data from Arabic. En Y. Suleiman (Ed.) Language and Society in the Middle East and North Africa. Londres: Curzon.

How to cite this paper:

Asensio Pastor, M. I. (2020). La mujer y el personaje femenino en cuentos jordanopalestinos de tradición oral. Journal of Literary Education, (3), pp. 112-136. doi: 10.7203/JLE.3.18950 